\title{
Aprendizagem Discriminativa Após Choques Incontroláveis ${ }^{1}$
}

\author{
Angélica Capelari \\ Universidade Metodista de São Paulo \\ Maria Helena Leite Hunziker \\ Universidade de São Paulo
}

\begin{abstract}
RESUMO
O objetivo desse estudo foi investigar se estímulos aversivos não contingentes (incontroláveis) produzem interferência em um processo de aprendizagem discriminativa reforçada positivamente. Vinte e quatro ratos foram distribuídos em três grupos $(n=8)$ que diferiram entre si quanto ao tratamento recebido na primeira sessão: choques controláveis (C), incontroláveis (I) ou nenhum choque $(\mathrm{N})$. Posteriormente, todos foram submetidos a 10 sessões de treino discriminativo com reforço positivo, em esquema múltiplo-concorrente FR-6/Extinção. Os resultados do treino discriminativo mostraram que, na primeira sessão, a exposição prévia a choques produziu menor frequência de respostas, sendo esse efeito levemente mais acentuado no grupo I; na décima sessão, os grupos não diferiram entre si quanto à frequência de respostas e ao índice discriminativo, que ficou acima de 0,80 . O efeito transitório do tratamento com choques incontroláveis, sem interferência a longo prazo no estabelecimento do controle de estímulos por reforçamento positivo, é contrário à generalidade do desamparo aprendido para contextos não aversivos.
\end{abstract}

Palavras-chave: desamparo aprendido; incontrolabilidade; controle de estímulos.

\section{ABSTRACT \\ Discriminative Learning After Uncontrollable Shocks}

This experiment investigated whether non-contingent (uncontrollable) aversive stimuli can produce interference on subsequent positively reinforced discriminative learning. In the first session, rats were divided into three groups $(\mathrm{n}=8)$ exposed to controllable $(\mathrm{C})$, uncontrollable (I) or no-shock $(\mathrm{N})$ conditions. After that, all rats were exposed to 10 sessions of positive reinforcement under a multipleconcurrent FR 6/Extinction. The results showed a transitory effect: in the first session of the discriminative training, previous exposure to shocks reduced response frequency, and this suppressive effect was slightly stronger for group I; in the tenth session, all groups showed high response frequency and equal discriminative indexes (higher than 0,80). These results suggest that uncontrollable aversive stimuli do not interfere on positively reinforced discriminative learning, contrary to a generalized learned helplessness effect between aversive and appetitive contexts.

Keywords: learned helplessness; uncontrollability; stimulus control.

Diversos estudos têm demonstrado que indivíduos submetidos a choques elétricos independentes do seu comportamento (choques incontroláveis) apresentaram, posteriormente, dificuldade de aprender respostas de fuga, efeito esse denominado desamparo aprendido (Seligman, \& Maier, 1967). O fato de que um efeito similar não é obtido após choques controláveis tem apontado para a incontrolabilidade do estímulo como a variável crítica para a produção do desamparo (Peterson, Maier \& Seligman, 1993; Seligman, Maier \& Solomon, 1971).

Apesar de esses estudos terem sido, em sua maioria, realizados com choques elétricos, a análise do desamparo aprendido vem sendo generalizada para diversos contextos que envolvem outros estímulos, aversivos ou não, sendo bastante divulgada a sua proposição como modelo animal de depressão humana, a qual pressupõe essa generalidade (Peterson e cols., 1993; Seligman, 1975). No laboratório animal, dificuldades técnicas na utilização de outros estímulos aversivos, além do choque elétrico, têm limitado a investigação da generalidade do efeito para outros contextos aversivos. Apenas recentemente foram divulgados resultados consistentes demonstrando a eficácia do jato de ar quente (JAQ) como estímulo punidor (Carvalho Neto e cols., 2005; Carvalho Neto, Maestri \& Menezes, 2007), como reforçador negativo 
(em contingências de fuga) e como produtor do desamparo aprendido em condições onde a sua liberação independente do comportamento do sujeito (Maestri, 2008). Esses dados ampliam a generalidade do desamparo aprendido dentro de contextos aversivos, expandindo as relações anteriormente identificadas basicamente com choques elétricos.

O mesmo não se dá, contudo, quanto à investigação da generalidade do desamparo aprendido entre os contextos aversivo e apetitivo, ou seja, se a exposição à incontrolabilidade em um desses contextos produz $\mathrm{o}$ desamparo aprendido testado em outro. Tecnicamente, é possível investigar se choques elétricos incontroláveis interferem em aprendizagens de respostas reforçadas positivamente, ou se estímulos apetitivos incontroláveis interferem em aprendizagens de respostas reforçadas negativamente. Apesar de tecnicamente possível, esse tipo de investigação tem gerado resultados contraditórios. Alguns estudos mostram que estímulos apetitivos incontroláveis dificultaram a aprendizagem frente ao reforço negativo (Goodkin, 1976; Sonoda, \& Hirai, 1992) e que choques incontroláveis reduziram a aprendizagem reforçada positivamente (Calef, Choban, Shaver, Dye \& Geller, 1986). Porém, ao contrário, outros estudos não mostraram generalidade entre contextos: choques incontroláveis não afetaram aprendizagem reforçada positivamente (Rapaport \& Maier, 1978; Rosellini, 1978), e eventos apetitivos incontroláveis não interferiram na posterior aprendizagem de fuga (Beatty \& Maki, 1979).

A grande diversidade de procedimentos utilizados nesses estudos pode ser a responsável pelos resultados divergentes. Alguns procedimentos promovem a introdução de variáveis não previstas no estudo do desamparo, dificultando a análise dos resultados obtidos, uma vez que mesclam controles operantes e respondentes que controlam comportamentos antagônicos (ver Hunziker \& Santos, 2007, para uma análise mais detalhada sobre essa questão). Utilizando o procedimento proposto por Hunziker (1982), que estabelece com precisão o controle operante da resposta de fuga, Capelari e Hunziker (2005) obtiveram que ratos aprenderam normalmente essa resposta apesar de terem sido previamente expostos à liberação de água independente do comportamento. Esse resultado fortalece a indicação de que eventos apetitivos incontroláveis não produzem, posteriormente, desamparo aprendido em contexto aversivo.

O objetivo do presente estudo é ampliar essa investigação sobre a ocorrência do desamparo aprendido manipulando-se estímulos aversivos e apetitivos nas diferentes fases experimentais. Especificamente, esse estudo visa verificar se eventos aversivos incontroláveis interferem em aprendizagem operante reforçada positivamente.

\section{MÉTODO}

\section{Sujeitos}

Os sujeitos foram 24 ratos albinos, machos, Wistar, experimentalmente ingênuos, de aproximadamente 120 dias de idade, provenientes do Biotério da Faculdade de Psicologia da Fundação Padre Anchieta. Os sujeitos foram mantidos em gaiolas individuais por, no mínimo, uma semana antes de iniciar o experimento, com alimento (ração seca, balanceada) e água sempre disponíveis. Uma semana antes do início da coleta de dados, os animais foram privados de água através do esquema de acesso à água por 6 minutos/dia, sendo que, nos dias em que houve sessão experimental, os animais receberam 3 minutos de acesso à água após a sessão. Todos os sujeitos foram pesados diariamente para controlar as suas condições de saúde. Os procedimentos experimentais foram realizados durante o período de luz do ciclo de 12 horas luz/escuro (7:00 às 19:00).

\section{Equipamento}

Foram utilizadas duas caixas com focinhadores, nas quais os choques elétricos eram liberados através do piso, e três caixas com barras como manipulandum, para reforçamento positivo. As caixas com os focinhadores mediam 21,5 x 21,5 x 21,0 cm (comprimento, largura e altura), com a parede frontal de acrílico transparente e as demais em alumínio. No centro da parede lateral direita havia um orifício de $3,0 \mathrm{~cm}$ de diâmetro localizado a $6,0 \mathrm{~cm}$ acima do piso. Esse orifício se conectava a uma cuba medindo $14,0 \times 6,0 \mathrm{x}$ $9,0 \mathrm{~cm}$ (comprimento, largura e altura), na qual havia, a 1,5 cm da borda, uma célula foto-elétrica acionada por um feixe de luz infravermelha localizado no lado oposto a ela. A introdução de qualquer objeto na cuba, por um mínimo de $1,5 \mathrm{~cm}$ de profundidade, interrompia esse feixe de luz, registrando uma resposta. Nesse caso, geralmente os animais introduzem seus focinhos, originando a chamada resposta de focinhar. As caixas para reforçamento positivo mediam $20,0 \times 23,5$ x $22,0 \mathrm{~cm}$ (profundidade, largura e altura respectivamente), sendo o piso composto por barras cilíndricas de $0,3 \mathrm{~mm}$ de diâmetro, distando $1,3 \mathrm{~cm}$ entre si. As duas paredes laterais e o fundo eram de alumínio, sendo a parede frontal e o teto de acrílico. Na parede lateral direita havia duas barras cilíndricas de latão 
medindo 4,0 cm de comprimento e $0,5 \mathrm{~cm}$ de diâmetro, equidistantes do centro da parede, afastadas $10,5 \mathrm{~cm}$ entre si (centro a centro) e $8,5 \mathrm{~cm}$ acima do piso. Uma pressão de no mínimo 45,0 gf (grama-força) em cada uma dessas barras produzia o acionamento de um microswitch localizado do lado externo da caixa, o qual era acompanhado de um ruído audível tipo "clique". O acionamento do microswitch era registrado automaticamente, caracterizando a emissão de uma resposta de pressão à barra. Ambas as barras podiam ser removidas, permitindo que, em parte do experimento, a caixa permanecesse com apenas uma delas. No centro dessa parede, equidistante das barras, e ao nível do piso, havia um bebedouro com capacidade de liberar $0,05 \mathrm{cc}$ de água, que correspondia a um reforço. A 7,0 cm acima de cada barra havia uma lâmpada tipo "olho de boi".

Todas as caixas eram individualmente isoladas, visual e acusticamente, por câmaras construídas em compensado e fórmica. Visores do tipo "olho mágico", ou recortes em acrílico na tampa frontal das caixas de isolamento, permitiam a observação dos sujeitos durante as sessões. Um exaustor localizado na parede lateral direita da câmara de isolamento produzia a renovação do ar e um ruído constante que abafava ruídos externos.

A umidade relativa do ar foi controlada através de um higrômetro localizado dentro da sala de coleta de dados. Ela foi mantida abaixo de $70 \%$, e ultrapassando esse limite, era acionado um desumificador (Marca Arsec Modelo 160M3-U). Controles e registros foram realizados por computadores que se localizavam em salas adjacentes à de coleta de dados. Na fase de tratamento, utilizou-se um computador PC 386 com programa escrito em linguagem Delphi e na fase de tratamento, foi utilizado um computador Machintosh Color Classic com software escrito em linguagem True Basic, ambos especialmente desenvolvidos para esse experimento.

\section{Procedimento}

Antes de iniciar o experimento todos os sujeitos passaram pelo procedimento de modelagem, por aproximações sucessivas, da resposta de pressionar a barra. Foram realizadas duas sessões, nas quais uma das barras foi retirada da caixa experimental: na primeira sessão, foi modelada a resposta de pressão à barra esquerda, e na sessão seguinte, a resposta de pressão à barra direita. Em ambas as sessões, a modelagem foi seguida por 100 reforços em CRF.
Após essa fase preliminar, os sujeitos foram distribuídos em três grupos $(n=8)$ considerando-se seus pesos corporais, distribuídos equitativamente entre os grupos. O experimento constou de duas fases (tratamento e teste), sendo os grupos diferentes entre si quanto ao tratamento recebido: choque controlável (C), choque incontrolável (I) ou nenhum choque (N). Os sujeitos do Grupo C foram colocados na caixa com focinhador onde, após $50 \mathrm{~s}$, receberam 60 choques elétricos, não sinalizados, de $1 \mathrm{~mA}$, ministrados a intervalos variáveis de 60s (amplitude de variação 10-110 s). A introdução do focinho do sujeito na cuba da parede desligava imediatamente o choque, sendo essa resposta chamada de "focinhar". Cada choque correspondeu a uma tentativa, sendo que o tempo decorrido entre o início e o término do choque foi registrado como a latência da resposta na tentativa. Não havendo a resposta de focinhar, o choque era interrompido automaticamente após $10 \mathrm{~s}$ do seu início. Tentativas onde ocorreram latências de $10 \mathrm{~s}$ foram registradas como tentativas falhas. Respostas emitidas no intervalo entre choques não foram registradas e nem tiveram consequência programada. Cada sujeito do Grupo $\mathrm{C}$ foi acoplado a um sujeito do Grupo I de forma que ambos recebiam choques simultaneamente. Assim, quando o choque era interrompido para o animal do Grupo C, ele também o era para o animal do Grupo I a ele acoplado, cujas respostas não tinham consequência programada. Portanto, na fase de tratamento, os sujeitos desses dois grupos foram submetidos a uma sessão na qual receberam igual frequência e distribuição de choques, sendo a única diferença entre eles que os sujeitos do Grupo C podiam desligar os choques emitindo a resposta de focinhar, enquanto que os do Grupo I tinham o choque desligado independente da emissão de qualquer resposta sua. Os animais do grupo restante $(\mathrm{N})$ não receberam tratamento, sendo mantidos no biotério durante essa fase do experimento.

Ao término da sessão de choques, os detritos deixados pelo sujeito nas caixas experimentais eram retirados, sendo as mesmas limpas com um pano umedecido em água e, em seguida, outro pano umedecido com álcool. Esse procedimento visou reduzir os estímulos olfativos deixados pelos sujeitos submetidos aos choques, uma vez que já foi demonstrado que ratos produzem odores específicos quando expostos à situação aversiva (Bolles, 1970). A presença de tais odores poderia sinalizar situação aversiva para os ratos a serem colocados nessa caixa experimental, interferindo nos dados coletados.

A fase de teste teve início $24 \mathrm{~h}$ após a sessão de tratamento, sendo todos os animais igualmente sub- 
metidos ao procedimento que segue. Na caixa com duas barras os sujeitos foram submetidos a 10 sessões em esquema de reforço múltiplo-concorrente FR/extinção. Nesse esquema, ambas as barras permaneciam constantemente disponíveis, cada uma relacionada a um esquema de reforço sinalizado: luz acesa sobre a barra (estímulo discriminativo ou SD) era correlacionada com reforço liberado em esquema FR, enquanto que luz apagada (estímulo delta ou $\mathrm{S} \Delta$ ) era correlacionada com ausência de reforço (extinção). Os esquemas se alternavam entre as barras de forma que os reforços foram liberados, ora contingentes apenas às respostas na barra direita e ora apenas às respostas na barra esquerda. Cada condição de estímulo durava $60 \mathrm{~s}$, período esse chamado de componente do esquema. As sessões tiveram duração fixa (50 min), com 25 componentes de FR em cada barra. A alternação dos componentes entre barras se deu de forma aleatória, com o limite de não haver mais do que três componentes iguais apresentados sucessivamente em uma mesma barra. A mudança da contingência de reforçamento para a barra alternativa cancelava as respostas emiti- das na barra anterior que não haviam completado a razão exigida. Portanto, ao retornar o reforço a essa barra, tais respostas não eram consideradas para o atendimento da contingência. Na Sessão 1, o esquema de reforço utilizado foi FR3/extinção, passando a FR6/extinção nas sessões sucessivas, caso o sujeito recebesse, no mínimo, 50 reforços na primeira sessão. No caso de o critério não ter ser atingido, uma nova sessão de FR3/extinção era realizada até que o critério fosse atendido e a sessão seguinte passasse a FR6/extinção.

Foram registradas separadamente as frequências de respostas em cada barra (D e E), frente ao $\mathrm{SD}$ e ao $\mathrm{S} \Delta$, e os reforços recebidos. A aprendizagem do sujeito quanto ao controle de estímulos estabelecido foi avaliada através do índice discriminativo (ID), calculado a partir da razão entre número de respostas dadas na barra sob reforçamento (respostas corretas) e o número de respostas totais da sessão.

A Tabela 1 resume o delineamento utilizado neste estudo.

TABELA 1

Grupos Experimentais Compostos em Função dos Diferentes Tratamentos Recebidos na Fase de Tratamento

\begin{tabular}{|c|c|c|}
\hline Grupos & TRATAMENTO (1 sessão) & TESTE (10 sessões) \\
\hline \multirow{4}{*}{ C } & 60 choques controláveis & \\
\hline & $\mathrm{R}$ de fuga $=$ focinhar & Esquema múltiplo/concorrente \\
\hline & $1,0 \mathrm{~mA}$ & $S D=\operatorname{luz}$ \\
\hline & máx. 10s & $\mathrm{S} \Delta=$ escuro \\
\hline \multirow{4}{*}{ I } & 60 choques incontroláveis & $\mathrm{R}=$ pressão à barra (D ou E) \\
\hline & $1,0 \mathrm{~mA}$ & Reforço positivo = água \\
\hline & máx $10 \mathrm{~s}$ & Sessão 1 - FR3/Ext. \\
\hline & (Acoplado) & Sessões 2 a 10 - FR6/ Ext. \\
\hline $\mathbf{N}$ & -------------------- & \\
\hline
\end{tabular}

A análise estatística foi realizada utilizando o teste de Análise de Variância simples (ANOVA one-way) e para medidas repetidas (ANOVA two-way). Quando necessário, as diferenças entre duplas foram analisadas pelo teste Scheffé ou Bonferroni. Na fase de teste foi utilizado o teste $t$ de Student para amostras relacionadas. Todos os resultados foram considerados estatisticamente significantes para um mínimo de $\mathrm{p}<0.05$.

\section{RESULTADOS}

$\mathrm{Na}$ fase de tratamento a resposta de focinhar foi emitida de forma diferenciada em função do tratamento recebido previamente. A Figura 1 mostra a frequência de emissão da resposta de focinhar durante os choques, apresentada pelos sujeitos dos grupos $\mathrm{C}$ e I ao longo de blocos de cinco tentativas sucessivas. Esses resultados mostram que o reforçamento negativo produziu um padrão sistemático de respostas emitidas com frequência crescente ao longo da sessão, chegando ao máximo de frequência possível (40 respostas) nos blocos finais. Os sujeitos expostos aos choques incontroláveis tiveram um padrão irregular, com ausência de respostas em alguns blocos e um máximo de sete respostas ao longo dos blocos finais. Essas diferenças entre os grupos se mostraram estatisticamente significantes $(F(1,14)=146,847 ; p<0,001)$. 
Grupo C

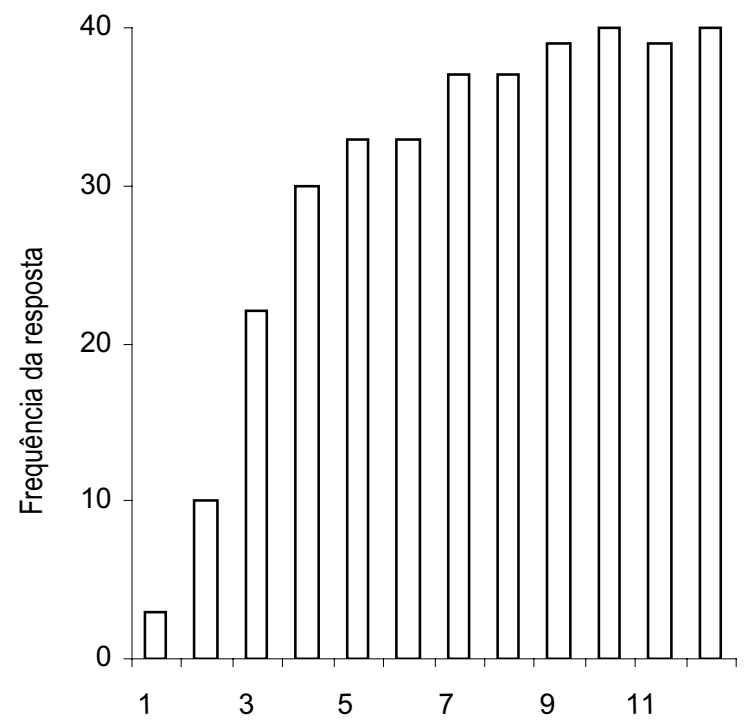

Grupo I

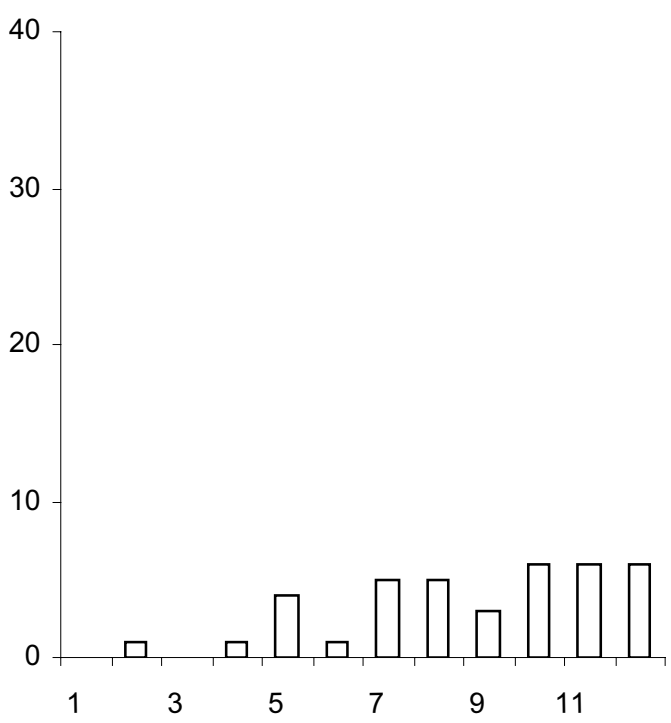

Blocos de 5 tentativas

Figura 1. Frequência de emissão da resposta de focinhar durante os choques, apresentada pelos sujeitos dos grupos $\mathrm{C}$ e I, em blocos de cinco tentativas.

Com relação à fase de teste, a Figura 2 mostra que os grupos diferiram acentuadamente na primeira sessão (parte superior da Figura), onde os sujeitos não expostos previamente a qualquer tratamento (Grupo $\mathrm{N}$ ) emitiram respostas em maior frequência do que os sujeitos dos dois grupos tratados com choques. Dentre estes, os animais submetidos previamente a choques incontroláveis foram os que apresentaram menor frequência da resposta reforçada positivamente. Quanto ao desempenho frente ao SD ou $\mathrm{S} \Delta$ (barras brancas e pretas, respectivamente), os sujeitos do grupo $\mathrm{N}$ apresentaram, já na primeira sessão, maior frequência de respostas na barra na qual o $\mathrm{SD}$ estava em vigor. $\mathrm{O}$ mesmo não ocorreu com os demais grupos, que mostram um responder indiferenciado, com semelhante frequência frente a ambos os estímulos. Na décima sessão (parte inferior da Figura 2), os três grupos apresentaram um desempenho muito próximo entre si: todos aumentaram a frequência total de respostas em SD (comparativamente à primeira sessão), com diferenças menos acentuadas frente a $\mathrm{S} \Delta$. Se comparados isoladamente os desempenhos frente a cada estímulo, verifica-se que os três grupos tiveram desempenhos praticamente iguais frente ao $\mathrm{S} \Delta$, mas mostraram diferenças frente ao SD: sob essa condição de estímulo, os animais do grupo I foram os que apresentaram maior frequência de respostas, enquanto que os do grupo $\mathrm{C}$ foram os que emitiram a resposta com menor frequência. A análise estatística da Sessão 1 mostra que houve diferença entre os grupos quanto ao total de respostas emitidas $(F(2,23)=22,844 ; p<0,001)$, sendo que essa diferença situou-se entre o grupo $\mathrm{N}$ e os demais $(\mathrm{p}<0,05)$, que não diferiram entre si. Quanto ao desempenho frente ao estímulo presente (SD ou $\mathrm{S} \Delta$ ), os grupos diferiram significantemente somente na primeira sessão $(\mathrm{F}(2,23)=19,006 ; \mathrm{p}<0,001)$ e $(\mathrm{F}(2$, $23)=104,476 ; p<0,001)$ respectivamente. Na sessão 10 , não foram observadas diferenças no total de respostas emitidas pelos sujeitos de todos os grupos. As diferenças do desempenho frente ao SD e $\mathrm{S} \Delta$ foram significantes em todos os grupos $(\mathrm{p}<0,001)$. 


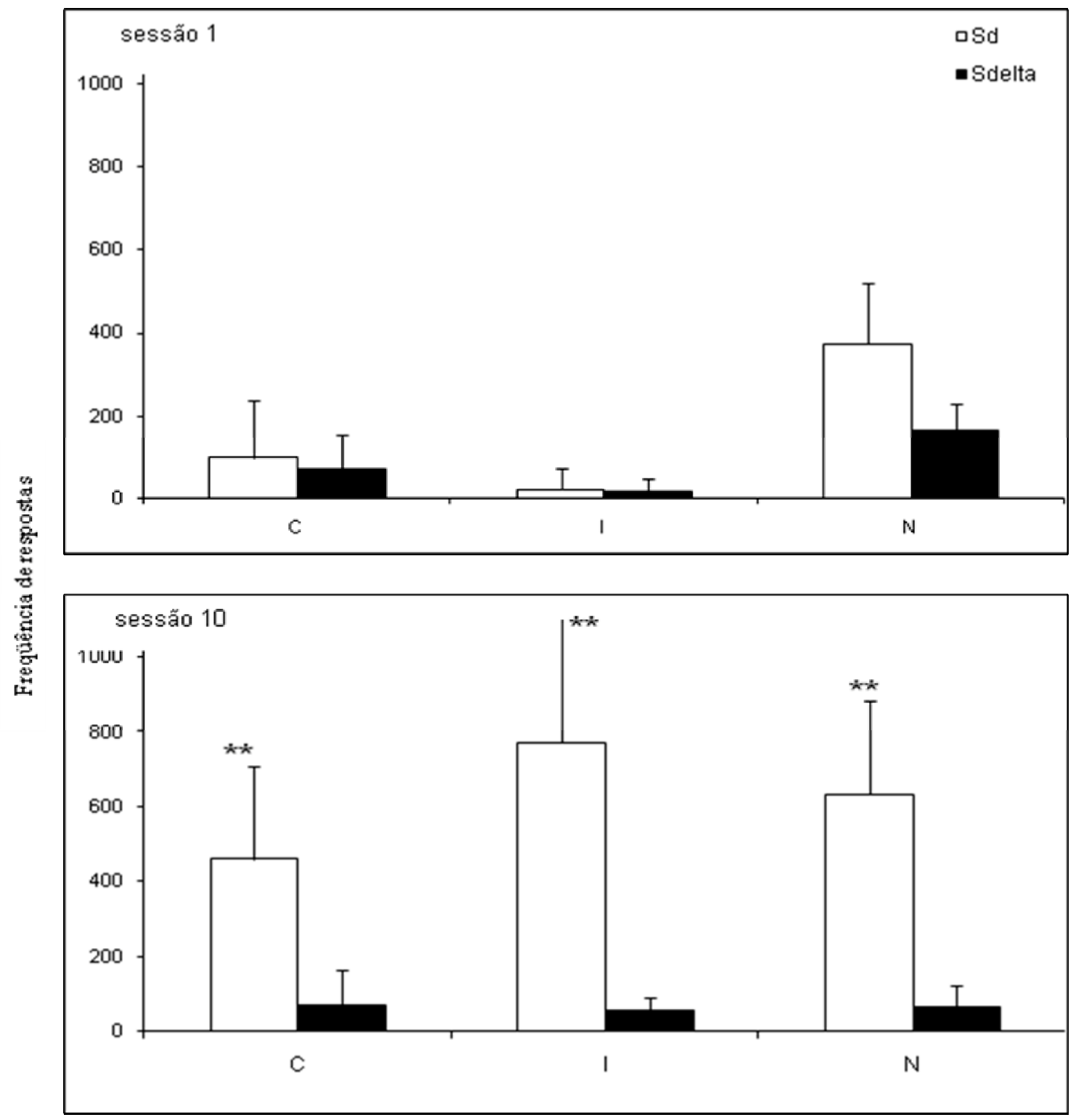

grupos

Figura 2. Frequência média de respostas de pressão à barra apresentadas pelos sujeitos dos Grupos Controlável (C), Incontrolável (I) e Nenhum choque (N), na primeira e na última sessão do teste de discriminação, nas condições de SD e S $\Delta$. $O$ asterisco indica que a diferença de desempenho nessas condições foi estatisticamente significante $\left(^{*}=p<0,05 e^{* *}=p<0,01\right)$.

A Figura 3 mostra o índice discriminativo médio apresentado pelos três grupos na primeira e última sessão do teste. Na primeira sessão os três grupos apresentaram desempenho pouco diferenciado em relação às duas condições de estímulo, emitindo cerca de $50 \%$ das respostas em cada alternativa. Os desempenhos extremos foram o do grupo I, que emitiu cerca de $40 \%$ das suas respostas frente ao SD e o do grupo $\mathrm{N}$, que emitiu aproximadamente $55 \%$ das respostas frente ao mesmo estímulo. Na última sessão, todos os três grupos apresentaram ID acima de 0,80 , sendo o índice mais alto obtido pelo grupo I. A análise estatística desses resultados mostrou que foram estatisticamente significantes as diferenças encontradas entre os 
grupos $(\mathrm{F}(2,20)=11,527 ; \mathrm{p}<0,001)$ e em relação à primeira e à décima sessões $(\mathrm{F}(1,20)=100,663$; $\mathrm{p}$ $<0,001)$, e que houve interação entre grupo e sessões $(\mathrm{F}(2,20)=9,863 ; \mathrm{p}<0,001)$. A comparação dos pares de grupos indicou que apenas o Grupo I diferiu dos demais na primeira sessão $(p<0,05)$, enquanto na décima sessão as diferenças entre os IDs não foram estatisticamente significantes. Comparando-se estatisticamente a diferença nos IDs de cada grupo em relação à primeira e a última sessão, todas foram significantes $(\mathrm{p}<0,05)$.

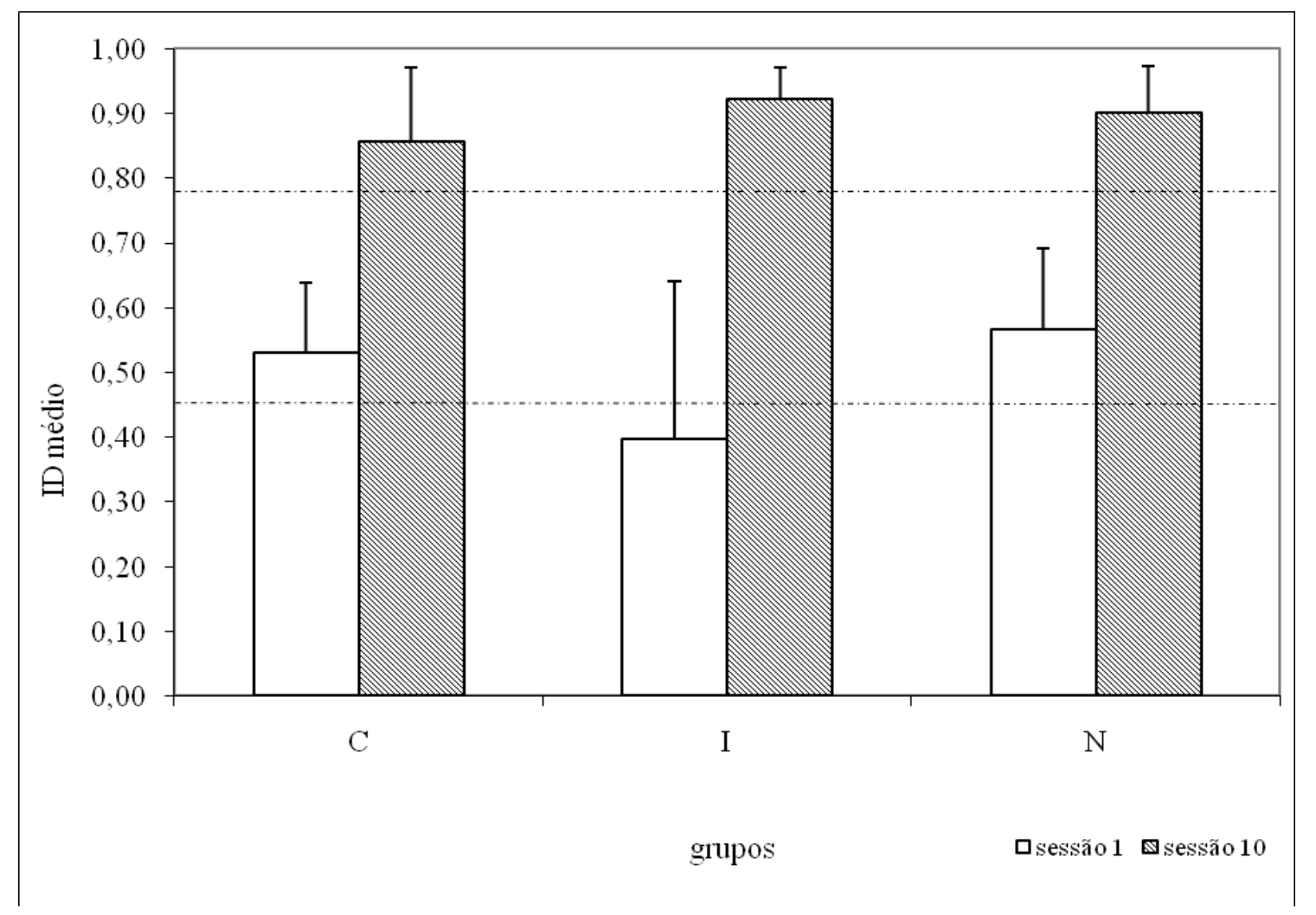

Figura 3. Índice discriminativo (ID) médio apresentados pelos sujeitos dos Grupos C, I e N, na primeira e na décima sessão do teste de discriminação.

\section{DISCUSSÃO}

$\mathrm{Na}$ fase de tratamento os animais do Grupo C emitiram com alta frequência a resposta que modificava a duração dos choques, tendo sido seu comportamento modificado por essa contingência de reforçamento negativo, ou seja, todos os sujeitos desse grupo apresentaram um aumento sistemático na emissão dessa resposta e diminuição das suas latências ao longo das sessões. Os animais do Grupo I, que previamente foram expostos a choques incontroláveis, não tiveram a resposta de focinhar selecionada, sendo as mesmas emitidas apenas esporadicamente com padrão irregular. Esses resultados mostram que o arranjo experimental estabelecido no presente estudo foi adequado para expor os sujeitos às variáveis independentes crí- ticas no estudo do desamparo aprendido, que são o controle ou a impossibilidade de controle sobre o término dos choques. Portanto, os resultados obtidos na fase de teste podem ser analisados em função dessas variáveis.

A análise dos efeitos das variáveis manipuladas difere se for considerada a primeira ou a última sessão do teste. Na primeira sessão ambos os grupos expostos a choques sofreram interferência na aquisição da resposta discriminativa, sendo esse efeito ligeiramente mais acentuado no grupo previamente tratado com choques incontroláveis; entretanto, na última sessão, esses animais se comportaram de forma semelhante aos animais ingênuos. $\mathrm{O}$ efeito alcançado na primeira sessão poderia sugerir que se tivesse obtido, mesmo que de forma pouco acentuada, uma replicação do 
desamparo aprendido. Porém, a interferência detectada no comportamento dos animais que tiveram controle sobre os choques enfraquece essa conclusão. É crítico nos estudos sobre o desamparo aprendido que a interferência ocorra apenas em função da incontrolabilidade dos choques e não dos choques em si (Maier \& Seligman, 1976; Seligman \& Maier, 1967). No presente estudo, o efeito do tratamento sobre o desempenho na primeira sessão foi, aparentemente, determinado mais pelos choques do que pela sua impossibilidade de controle.

$\mathrm{O}$ efeito indiferenciado dos dois grupos previamente expostos a choques, obtido na primeira sessão, pode ser atribuído a um controle respondente que tornou menos provável a emissão da resposta operante na condição de teste. Considerando que as caixas utilizadas na sessão de tratamento e nas sessões de teste tinham muitas semelhanças entre si (relativas ao material das paredes e piso, formato e tamanho, iluminação, ruído branco, etc), pode-se supor que o pareamento entre os estímulos físicos da caixa com focinhadora e os choques, na fase de tratamento, permitiu que a caixa adquirisse propriedades de estímulo aversivo condicionado (CS). Sabidamente, é menor a probabilidade de emissão de respostas frente ao CS aversivo, mesmo que positivamente reforçadas, como mostraram os estudos sobre supressão condicionada (Estes \& Skinner, 1941). Por outro lado, no decorrer do teste, o pareamento caixa/choque deixou de ocorrer, levando ao enfraquecimento da função de estímulo aversivo condicionado. Com a redução do controle aversivo do $\mathrm{CS}$, as respostas reforçadas positivamente se tornaram mais frequentes, o que permitiu aos sujeitos se exporem ao reforçamento positivo diferencial e aprenderem um responder discriminativo. Assim, mais do que um efeito de desamparo aprendido, que envolve redução de controle pelo reforço operante, é possível que o efeito visto nessa primeira sessão seja unicamente aliciado pelo CS aversivo, de forma indiferenciada nos Grupos I e C. Se o efeito na primeira sessão fosse, além de supostamente produzido pelo CS aversivo, também um efeito da insensibilidade à contingência operante, o esperado seria que no decorrer das sessões, quando o CS perdesse sua função aversiva, o grupo exposto a choques controláveis apresentasse desempenho equivalente ao dos animais ingênuos, mas os animais expostos à incontrolabilidade se mantivessem sem aprender. Não foi isso que se observou no atual estudo. A dissolução das diferenças entre grupos ao longo das sessões sugere, portanto, que não se obteve a insensibilidade à con- tingência operante, principal característica do desamparo aprendido.

Deve-se considerar que a exposição inicial do sujeito a uma condição de controle pode evitar o aparecimento do desamparo aprendido, efeito esse chamado de imunização (Hunziker \& Lima, 2006; Hunziker, Manfré \& Yamada, 2006; Seligman \& Maier, 1967; Seligman, Rosellini \& Kozak, 1975; Yano \& Hunziker, 2000). Assim, uma possibilidade a ser analisada é a de que a modelagem da resposta de pressão à barra, realizada no presente estudo antes da fase de tratamento com choques, pode ter imunizado os sujeitos em relação aos efeitos da incontrolabilidade dos choques, justificando a não obtenção do desamparo aprendido. De fato, os estudos sobre imunização têm demonstrado que esse efeito depende de alguns arranjos, além do fator controle versus não controle do estímulo. Aparentemente, a depender da natureza dos estímulos manipulados nas várias fases dos experimentos, é relevante a frequência de exposição aos estímulos controláveis que podem produzir a imunização. Por exemplo, Mestre e Hunziker (1996) obtiveram que uma sessão de modelagem com reforço positivo (como feito no presente estudo), antes do tratamento convencional com choques, não impediu o desenvolvimento do desamparo. Utilizando apenas estímulos aversivos em todas as fases, Yano e Hunziker (2000) mostraram que ratos expostos a uma única sessão de choques controláveis, seguida por uma de choques incontroláveis e teste de aprendizagem de fuga, não apresentaram desamparo (ou seja, apresentaram efeito de imunização). Esse processo de imunização foi, contudo, obtido com maior número de sessões de reforçamento positivo na primeira fase, seguidas por exposição a choques incontroláveis e teste de fuga (Hunziker e cols., 2006; Hunziker \& Lima, 2006). Levando-se em conta esses estudos, pode-se considerar pouco provável que uma única sessão de reforçamento positivo, ministrada antes da exposição aos choques incontroláveis, tenha promovido a imunização dos sujeitos em relação aos efeitos deletérios dos choques incontroláveis. Entretanto, como não há um estudo sistemático que investigue esse efeito de imunização utilizando reforçamento positivo na primeira e na última fase, intercaladas por choques elétricos incontroláveis, não se pode descartar totalmente a possibilidade de que a sessão de modelagem possa ter reduzido o efeito de desamparo aprendido que, caso contrário, poderia ser observado no teste de aprendizagem discriminativa. É preciso a realização de novo experimento, sem a modelagem inicial, para que tal hipótese seja testada. 
A transitoriedade do efeito da incontrolabilidade verificada no presente estudo é compatível com o descrito em outros experimentos. Com uma única sessão de teste, Rosellini (1978) observou que todos os sujeitos expostos previamente a choques incontroláveis mostraram dificuldade de aprendizagem nas tentativas iniciais, porém aprenderam a nova resposta operante (reforçada positivamente) se igualando aos demais grupos nas tentativas finais. Fazendo duas sessões de teste, Rosellini, DeCola, Plonsky, Warren e Stilman (1984) obtiveram que os desempenhos dos sujeitos dos três grupos diferiram inicialmente, mas se igualaram na segunda sessão. Por fim, realizando 10 sessões de teste, Rosellini, De Cola e Shapiro (1982) mostraram que os animais expostos a choques incontroláveis demoraram mais sessões para atingir o índice de discriminação (80\%) sob contingência de reforçamento positivo, porém igualaram seu desempenho aos dos demais sujeitos a partir da quarta sessão.

Esses testes com múltiplas sessões não são utilizados nos estudos de desamparo que envolvem apenas controle aversivo. No geral eles realizam apenas uma sessão no teste, na qual não se observa essa redução do efeito da incontrolabilidade. Ao contrário, diversos estudos têm demonstrado que a diferença obtida em função da incontrolabilidade dos choques é crescente ao longo da sessão, uma vez que os animais expostos a esse tratamento mantêm ou acentuam suas altas latências de fuga, enquanto que os demais sujeitos reduzem suas latências ao longo da sessão (Hunziker e cols., 2006; Hunziker \& Santos, 2007; Mestre \& Hunziker, 1996; Yano \& Hunziker, 2000).

Essa diferença de intensidade e transitoriedade do efeito entre os estudos de desamparo que utilizam apenas estímulo aversivo (choque) ou estímulos apetitivos (água) e aversivos (choque) precisa ser melhor investigada. É possível que o uso de estímulos similares em todas as fases amplie a generalização, para o teste, da aprendizagem de falta de controle, supostamente presente na fase de tratamento com eventos incontroláveis. Porém, ao se utilizarem estímulos de naturezas tão distintas quanto choque e água, os resultados obtidos no presente estudo indicam uma quase total ausência de efeito da incontrolabilidade, ou seja, sugerem a não generalidade do desamparo entre contextos. Esses resultados são consistentes com os obtidos em estudos com a condição invertida, onde o evento incontrolável apetitivo (água) não promoveu o desamparo aprendido sobre aprendizagem de fuga (Capelari \& Hunziker, 2005). No seu conjunto, portanto, alguns trabalhos sugerem uma baixa generali- dade do desamparo entre contextos aversivo e positivo, embora outros mostrem que o efeito de imunização se dá quando a natureza dos estímulos da primeira exposição de controle é diferente dos utilizados nas demais fases (Hunziker e cols., 2006; Hunziker \& Lima, 2006; Mestre \& Hunziker, 1996).

Por fim, pode-se sugerir que os resultados do presente estudo, adicionados a outros descritos na lieratura, sugerem que o efeito deletério da incontrolabilidade aversiva pode ser revertido pela oportunidade repetida dos sujeitos experimentarem o reforçamento positivo. Essa sugestão, compatível com uma proposta terapêutica, precisa ser mais explorada experimentalmente, pois tem consequências importantes para a compreensão do fenômeno do desamparo em si e para a sua interpretação como modelo experimental de depressão.

\section{REFERÊNCIAS}

Beatty, W. W., \& Maki, W. S. (1979). Acquisition of instrumental responding following non-contingent reinforcement: failure to observe "learned laziness" in rats. Bulletin of the Psychonomic Society, 13, 268-271.

Bolles, R. C. (1970). Species-specific defense reactions in avoidance learning. Psychology Review, 70, 32-48.

Calef, R. S., Choban, M. C., Shaver, J. P., Dye, J. D., \& Geller, E. S. (1986). The effect of inescapable shock on the retention of a previously learned response in an appetitive situation with delay of reinforcement. Bulletin of the Psychonomic Society, 24(3), 213-216.

Capelari, A., \& Hunziker, M. H. L. (2005). Desamparo aprendido em função de estímulos apetitivos incontroláveis. Psicologia: Teoria e Pesquisa, 21(1), 99-107.

Carvalho Neto, M. B., Maestri, T. C., Tobias, G. K. S., Ribeiro, T. C., Coutinho, E. C. M., Oliveira, R. C. V., Ferreira, F., Farias, D. C., \& Gomes, D. M. (2005). O jato de ar quente como estímulo punidor em rattus norvegicus. Psicologia: Teoria e Pesquisa, 21, 335-340.

Carvalho Neto, M. B., Maestri, T. C., \& Menezes, E. S. R. (2007). $\mathrm{O}$ jato de ar quente como estímulo aversivo: Efeitos de sua exposição prolongada em rattus norvegicus. Acta Comportamentalia, 14, 150-155.

Estes, W. K., \& Skinner, B. F. (1941). Some quantitative properties of anxiety. Journal of Experimental Psychology, 29(1), 390-340.

Goodkin, F. (1976). Rats learn the relationship between responding and environmental events: An expansion of the learned helplessness hypothesis. Learning and Motivation, 7, 382-393.

Hunziker, M. H. L. (1982). Considerações metodológicas sobre o estudo da incontrolabilidade. Psicologia, 8(3), 61-77.

Hunziker, M. H. L., \& Lima, R. S. G. G. (2006). Imunização ao desamparo aprendido após reforçamento positivo em ratos. Interação em Psicologia, 10(2), p. 195-206. 
Hunziker, M. H. L., Manfré, F. N., \& Yamada, M. T. (2006). Reforçamento positivo da variabilidade e da repetição imuniza contra o desamparo aprendido. Revista Brasileira de Análise do Comportamento, 2, 53-66.

Hunziker, M. H. L., \& Santos, C. V. (2007). Learned helplessness: Effects of response requirement and interval between treatment and testing. Behavioural Processes, 76(3), 183-191

Maestri, T. C. (2008). O estudo do desamparo aprendido em função de dois estímulos aversivos: Jato de ar quente e choque elétrico. Dissertação de mestrado não-publicada, Universidade de São Paulo.

Maier, S. F., \& Seligman, M. E. P. (1976). Learned helplessness: Theory and evidence. Journal of Experimental Psychology: General, 105(1), 3-46.

Mestre, M. B. A., \& Hunziker, M. H. L. (1996). O desamparo aprendido, em ratos adultos, como função de experiências aversivas incontroláveis na infância. Tuiuti: Ciência e Cultura, 6(2), 25-47.

Peterson, C., Maier, S. F., \& Seligman, M. E. P. (1993). Learned helplessness: A theory for the age of personal control. New York: Oxford University Press.

Rapaport, P. M., \& Maier, S. F. (1978). Inescapable shock and food-competition dominance in rats. Animal Learning and Behavior, 6, 160-165.

Rosellini, R. A. (1978). Inescapable shock interferes with acquisition of an appetitive operant. Animal Learning and Behavior, 6, 155-159.

Rosellini R. A., DeCola J. P., \& Shapiro (1982). Crossmotivational effects of inescapable shock are associative in nature. Journal of Experimental Psychology: Animal Behavior Process, 8(4) 376-388.
Rosellini R. A., DeCola, J. P., Plonsky, M., Warren, D. A., \& Stilman, A. J. (1984). Uncontrollable shock proactively increases sensitivity to response-reinforcer independence in rats. Journal of experimental Psychology: Animal Behavior Process, 10(3), 346-359.

Seligman, M. E. P., \& Maier, S. F. (1967). Failure to escape traumatic shock. Journal of Abnormal Psychology, 74(1), 1-9.

Seligman, M. E. P., Maier, S. F., \& Solomon, R. L. (1971). Unpredictable and uncontrollable aversive events. Em E. F. Bush (Org.). Aversive conditioning and learning. New York: Academic Press.

Seligman, M. E. P., Rosellini, R. A., \& Kozak, M. J. (1975). Learned helplessness in the rat: Time course, immunization, and reversibility. Journal of Comparative and Physiological Psychology, 88, 542-547.

Sonoda, A., \& Hirai, H. (1992). The role of predictability in preventing escapes deficits following loss of control over. Animal Learning and Behavior, 20, 427-430.

Yano, Y., \& Hunziker, M. H. L. (2000). Desamparo aprendido e "imunização" com diferentes respostas de fuga. Acta Comportamentalia, 8, 143-166.

\section{Nota:}

1 Esse trabalho é parte da dissertação de mestrado defendida pela primeira autora, na Universidade de São Paulo, em 2002, sob a orientação da segunda autora. Ele foi parcialmente apresentado na XXX Reunião Anual de Psicologia da Sociedade Brasileira de Psicologia, realizada em Brasília/DF em 2000, e encaminhado para a $30^{\text {th }}$ Annual ABA Convention, realizada em Boston (USA), em 2004. Apoio CNPq.

\section{Sobre as autoras:}

Angélica Capelari: Psicóloga, Mestre em Psicologia pela Universidade de São Paulo, professora da Universidade Metodista de São Paulo.

Maria Helena Leite Hunziker: Psicóloga, Doutora em Psicologia pela Universidade de São Paulo, Professora Livre Docente da Universidade de São Paulo.

Endereço para correspondência: Maria Helena Leite Hunziker - Universidade de São Paulo - Av. Prof. Mello Moraes, 1721 05508-900 São Paulo/SP - Endereço eletrônico: hunziker@lexxa.com.br. 